\title{
Use of quick sequential organ failure assessment score-based sepsis clinical decision support system may be helpful to predict sepsis development
}

\author{
Youn-Jung Kim ${ }^{1}$, Jae-Ho Lee ${ }^{1,2, \star}$, Sang-Wook Lee ${ }^{3}$, Won Young Kim ${ }^{1}$
}

\author{
${ }^{1}$ Department of Emergency Medicine, \\ Asan Medical Center, University of Ulsan \\ College of Medicine, 05505 Seoul, \\ Republic of Korea \\ ${ }^{2}$ Department of Information Medicine, \\ Asan Medical Center, University of Ulsan \\ College of Medicine, 05505 Seoul, \\ Republic of Korea \\ ${ }^{3}$ Medical Information Office, Asan \\ Medical Center, 05505 Seoul, \\ Republic of Korea \\ *Correspondence \\ jaeholee@amc.seoul.kr \\ (Jae-Ho Lee)
}

\begin{abstract}
Objectives: A sepsis clinical decision support system (CDSS) can facilitate quicker sepsis detection and treatment and consequently improve outcomes. We developed a qSOFA-based sepsis CDSS and evaluated its impact on compliance with a 3-hour resuscitation bundle for patients with sepsis.

Methods: This before-and-after study included consecutive adult patients with suspected infection and qSOFA scores of $\geq 2$ at their emergency department (ED) presentation of a tertiary care hospital. Sepsis was defined according to the Sepsis3 criteria. We evaluated the 3-hour resuscitation bundle compliance rate for control patients from July through August 2016, for patients using the qSOFA-based sepsis CDSS from September through December 2016, and the impact of the system using multivariable logistic regression analysis.

Results: Of 306 patients with suspected infection and positive qSOFA scores at presentation, 265 patients $(86.6 \%)$ developed sepsis (including 71 patients with septic shock). The 3-hour resuscitation bundle compliance rate did not differ significantly between the patients before and after the routine implementation of the qSOFA-based sepsis CDSS $(63.7 \%$ vs. $52.6 \% ; P=0.071)$. Multivariate analysis showed that age (AOR [adjusted odds ratio], 1.033; $P=0.002$ ) and body temperature (AOR, $1.677 ; P<$ $0.001)$ were associated with bundle compliance.

Conclusions: Among patients with a positive qSOFA score at presentation, sepsis developed in $86.6 \%$, which means the qSOFA-based sepsis CDSS may be helpful; however, it was not associated with improved bundle compliance. Future quality improvement studies with multifactorial, hospital-wide approaches using sepsis CDSS tools are warranted.
\end{abstract}

\section{Keywords}

Quick SOFA; Compliance; Resuscitation bundle; Clinical decision support system; Sepsis

\section{Introduction}

Despite recent medical advances, the morbidity and mortality rates associated with sepsis remain high. Early recognition and timely management of sepsis are key factors for improving outcomes [1,2]. In 2016, as part of the efforts to enhance the early recognition and timely intervention of sepsis management, a joint Society for Critical Care Medicine (SCCM) and European Society of Intensive Care Medicine (ESICM) task force newly defined sepsis as life-threatening organ dysfunction due to a dysregulated response, i.e., an increase of at least 2 points in the Sequential [Sepsis-related] Organ Failure Assessment (SOFA) score [1, 2]. Additionally, the quick Sequential Organ Failure Assessment (qSOFA) score, using respiratory rate, blood pressure, and mental status, was announced as a rapid bedside assessment tool for screening patients at risk of sepsis development [1, 2].

Surviving Sepsis Campaign (SSC) guidelines have also emphasised immediate resuscitation, in the form of 3-hour and 6-hour resuscitation bundles, and timely and aggressive resuscitation, achieved by compliance with 3-hour resuscitation bundles for every patient at risk of sepsis would improve outcomes [3-5].

A clinical decision support system (CDSS) offers systematic applications of health-related knowledge and analysis of available data directly to the clinician, facilitating key performance goals and adherence to best practices [6-8]. A study from the United States about electronic surveillance for systemic inflammatory response syndrome criteria and hypotension demonstrated the effectiveness of provider alerts for improving early goal-directed therapy [9]; however, there 


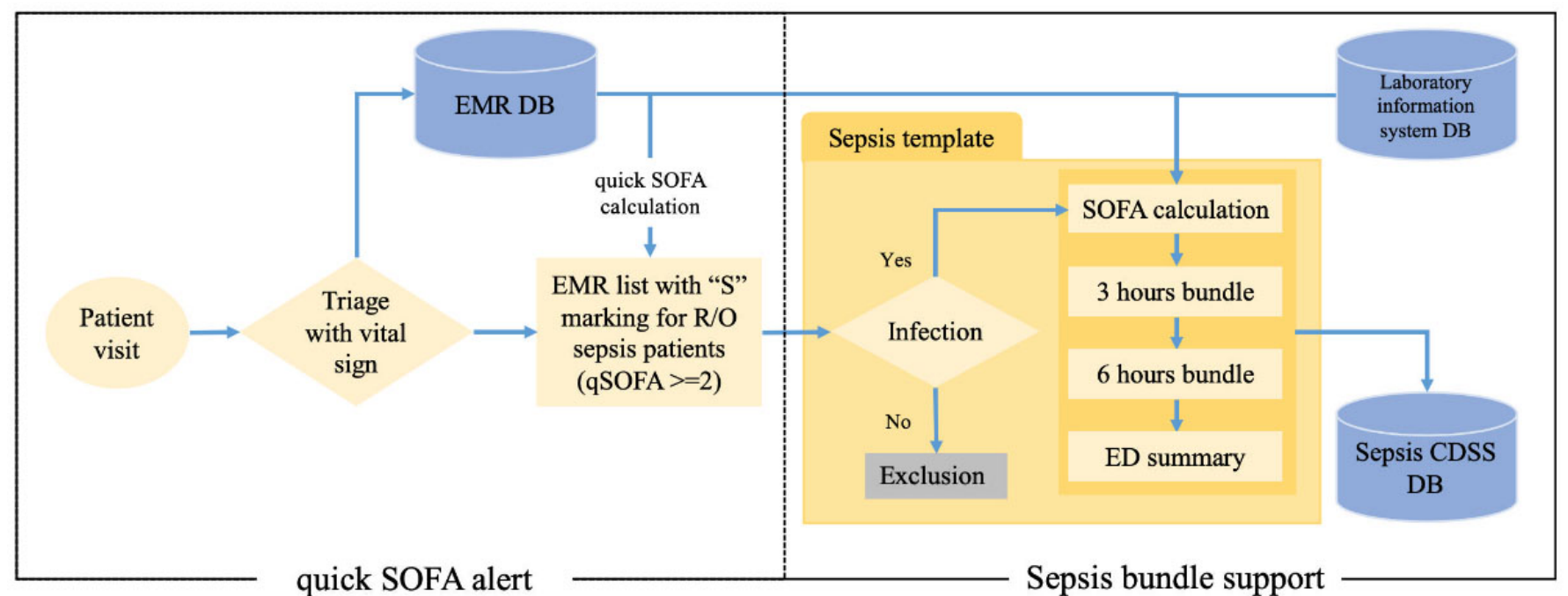

F I G U R E 1. Timeline of the quick Sequential Organ Failure Assessment (qSOFA) score-based sepsis clinical decision support system. CDSS, clinical decision support system; DB, data base; EMR, electronic medical records; qSOFA, quick Sequential Organ Failure Assessment.

is a paucity of sepsis CDSS data regarding to the new sepsis definition. Therefore, we developed a qSOFA score-based sepsis CDSS to help ED physicians and nurses promptly detect patients at high risk of sepsis and enhance compliance with 3-hour resuscitation bundles. In this before-and-after study, we evaluated the rate of sepsis and septic shock development among patients with qSOFA scores $\geq 2$ and the impact of the qSOFA score-based sepsis CDSS on compliance with a 3-hour resuscitation bundle for sepsis patients admitted to our ED. We hypothesised that the early recognition of patients at risk of sepsis development at the time of emergency department (ED) presentation would facilitate the implementation of 3hour resuscitation bundles.

\section{Methods}

Asan Medical Center's institutional review board approved the study (Study No. 2016-0818) and waived the requirement for informed consent because the study involved the analysis of a case registry. The research is in accordance with the Helsinki Declaration of 1975, as revised in 2010.

\subsection{Introduction to the qSOFA-based sepsis CDSS}

A team of emergency medicine and information medicine doctors and a technician from the medical information office developed a qSOFA score-based sepsis CDSS for ED physicians and nurses between January and July 2016. The system automatically calculated the qSOFA score using every patient's vital signs at ED presentation, and when the patients had a qSOFA score $\geq 2$, this was indicated with a label of " $\mathrm{S}$ " beside their names in the list of electronic medical records (EMRs) (Fig. 1). In addition to a systolic blood pressure $\leq 100 \mathrm{mmHg}$ and a respiratory rate $\geq 2$ breaths/min, mental status at ED presentation was assessed according to the Alert/responsive, Voice/responsive, Pain/responsive, Unresponsive (AVPU) scale; unalert patients were considered to have an altered mental status [1, 10]. The ED physicians were advised to evaluate the "S" patients as soon as possible, whether or not the patient was suspected of having an infection. The sepsis CDSS template was provided after the physician confirmed "suspected infection". The template contained the positive component of the qSOFA score, a semi-automatic calculation of the SOFA score, and a checklist for the 3-hour and 6-hour resuscitation bundles for sepsis (Supplementary Fig. 1). Lactic acid levels were checked using point-of-care blood gas analysis with the GEM PremierTM 3000 (Instrumentation Laboratory, Lexington, MA, USA) and recorded automatically in the CDSS template. The timing of accomplishment for each bundle element was recorded to enhance resuscitation bundle compliance. This system was finally adopted for our EMR system from September 2016 onwards.

\subsection{Study design and population}

This retrospective before-and-after study was performed at the ED of a tertiary referral hospital in Seoul, Korea, with 110,000 annual ED visits. Our hospital treated the patients with sepsis and septic shock using the SSC's 3-hour and 6hour resuscitation bundle guidelines [3, 11]. Asan Medical Center's institutional review board approved the study (Study No. 2016-0818) and waived the requirement for informed consent because the study involved the analysis of a case registry.

The patients with positive qSOFA scores enrolled from September through December 2016 were considered the treatment group (for whom the qSOFA-based CDSS was used), whereas the patients enrolled from July through August 2016 were considered the control group (for whom the qSOFAbased CDSS was not used). The qSOFA score-based sepsis CDSS prospectively screened all consecutive adult patients (aged $>18$ years) with qSOFA scores $\geq 2$ at ED presentation, and an ED physician on duty confirmed if patients were suspected of having an infection or not. Patients with suspected infection and positive qSOFA scores were automatically regis- 


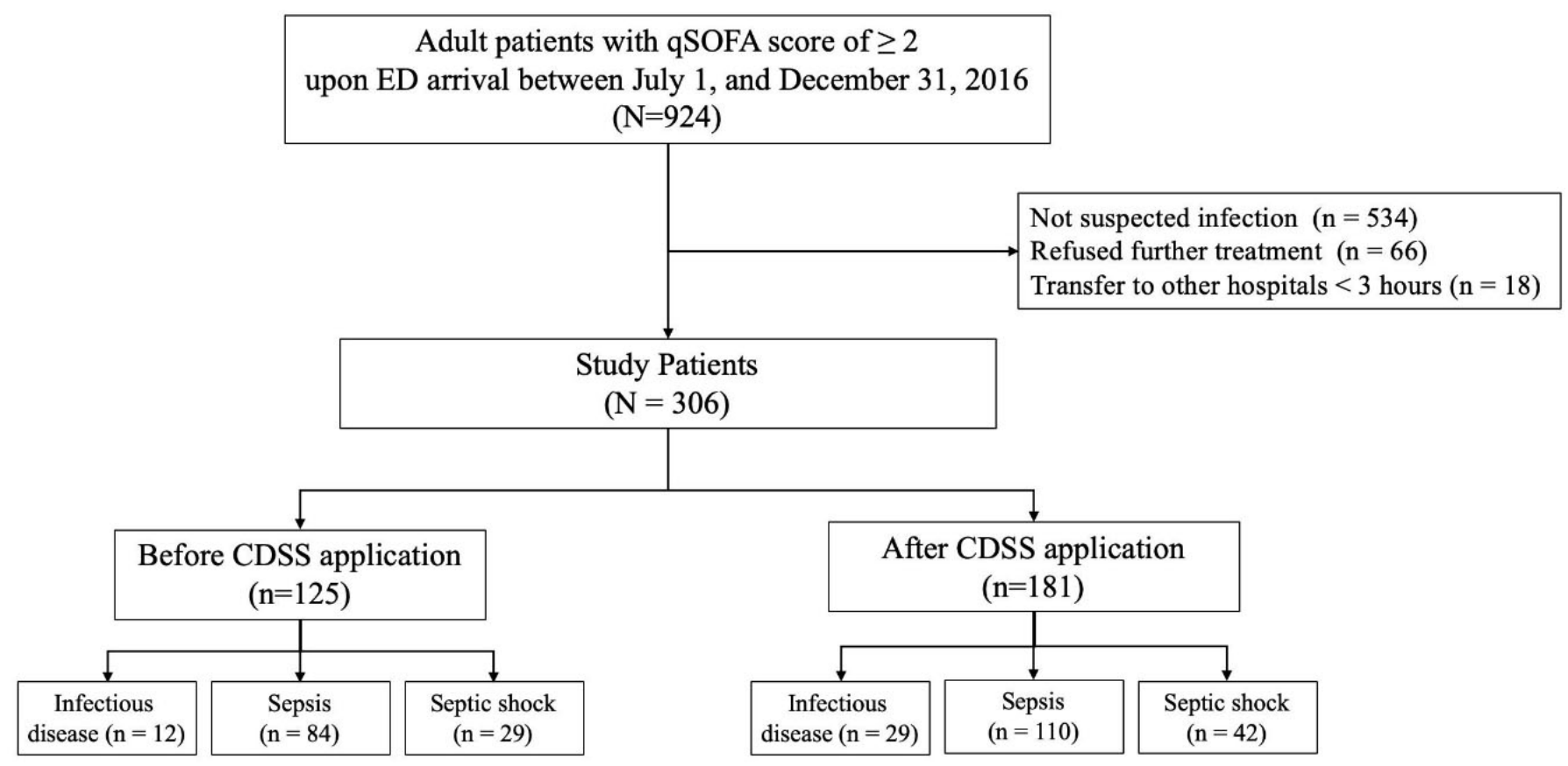

F I G U RE 2. Patient flow diagram. CDSS, clinical decision support system; ED, emergency department; qSOFA, quick Sequential Organ Failure Assessment.

tered in the qSOFA score-based sepsis CDSS patient list. We retrieved the data of patients with positive qSOFA scores for the control group, and an investigator (YJK) retrospectively reviewed and recorded their clinical data using the CDSS.

The study included all consecutive adult patients $(\geq 18$ years) with qSOFA scores $\geq 2$ at ED presentation with suspected or confirmed infection between July 1 and December 31, 2016. Patients were excluded from this study if they refused further examination and treatment; consented to a "do not resuscitate" order; or were directly transferred to another hospital from the ED within 3 hours. According to the Third International Consensus for sepsis and septic shock (Sepsis-3), we diagnosed the patients as having infectious disease, sepsis, and septic shock [2]. Patients with an acute increase of $\geq 2$ SOFA points were diagnosed with sepsis, and those who had persistent hypotension requiring vasopressors and serum lactate concentrations $>2 \mathrm{mmol} / \mathrm{L}$ after adequate fluid administration were diagnosed with septic shock [2].

The primary outcome was the completion of all 3-hour resuscitation bundle compliance for patients diagnosed with sepsis or septic shock during ED stay. The 3-hour resuscitation bundle includes (1) measuring lactate level; (2) obtaining blood cultures before antibiotic administration; (3) administering broad-spectrum antimicrobial agents; and (4) rapidly administering at least $30 \mathrm{~mL} / \mathrm{kg}$ of intravenous crystalloid for hypotension or lactate $\geq 4 \mathrm{mmol} / \mathrm{L}$ within 180 minutes after ED presentation. Our institution recommended administering the bolus of 200 to $300 \mathrm{~mL}$ crystalloid with close monitoring for patients with heart failure or end-stage renal disease, and $20 \mathrm{~mL} / \mathrm{kg}$ of intravenous crystalloid administration was recommended for those patients.

\subsection{Data collection}

For each patient, we captured the demographic information, vital signs at ED presentation, suspected or confirmed infection focus, baseline SOFA score, SOFA score at ED presentation, delta SOFA score, initial lactic acid, outcome, and the timing of 3-hour resuscitation bundle implementation recorded in the CDSS.

'Time zero' was defined as the ED presentation time, as listed on each patient's EMR [12]. The timing was recorded on the CDSS when each element of the resuscitation bundle was completed. In addition to the data retrieved from CDSS, the Charlson Comorbidity Index (CCI) and the mean ED occupancy rate for 3 hours after ED presentation were calculated; both of these values can affect compliance and outcomes [13, 14]. The CCI was used to assess the burden of chronic disease [13]. The ED occupancy rate was calculated as the total number of patients in our ED's critical care zone at each hour divided by the number of beds in the zone $[14,15]$. All of the patients were followed for 30 days after ED presentation.

\subsection{Statistics}

Continuous variables are expressed as means with standard deviation or medians with interquartile ranges due to their distribution, determined using the Kolmogorov-Smirnov test. Categorical data are presented as absolute numbers and percent frequencies. Student's $t$-test was used to compare the values of normally distributed continuous variables. The KruskalWallis rank test for 3 groups or the Mann-Whitney U test for 2 groups was used to compare skewed distributed continuous variables' values. Differences between categorical variables were analysed using the chi-square test or Fisher's exact test, as appropriate. The associations of the baseline characteristics and CDSS implementation with the 3-hour resuscitation 
TA B L E 1. The baseline characteristics of the study patients according to disease severity.

\begin{tabular}{|c|c|c|c|c|c|}
\hline Characteristic & Total $(\mathrm{n}=306)$ & Infectious disease $(n=41)$ & Sepsis $(n=194)$ & Septic shock $(n=71)$ & $P$-value \\
\hline Age, years & $68.0(57.0-77.0)$ & $60.0(41.5-76.0)$ & $68.5(56.0-77.0)$ & $69.0(61.0-78.0)$ & 0.04 \\
\hline Male & $174(56.9 \%)$ & $18(43.9 \%)$ & $115(59.3 \%)$ & $41(57.7 \%)$ & 0.19 \\
\hline Charlson Comorbidity Index & $5.0(3.0-7.0)$ & $4.0(2.0-7.0)$ & $5.5(3.0-7.0)$ & $5.0(4.0-7.0)$ & 0.14 \\
\hline qSOFA score & & & & & 0.003 \\
\hline 2 & $271(88.6 \%)$ & $39(95.1 \%)$ & $177(91.2 \%)$ & $55(77.5 \%)$ & \\
\hline 3 & $35(11.4 \%)$ & $2(4.9 \%)$ & $17(8.8 \%)$ & $16(22.5 \%)$ & \\
\hline \multicolumn{6}{|l|}{ qSOFA component } \\
\hline Respiratory rate $\geq 22 / \mathrm{min}$ & $262(85.6 \%)$ & $40(97.6 \%)$ & $170(87.6 \%)$ & $52(73.2 \%)$ & 0.001 \\
\hline $\begin{array}{l}\text { Systolic blood pressure }< \\
100 \mathrm{mmHg}\end{array}$ & $231(75.5 \%)$ & $30(73.2 \%)$ & $135(69.6 \%)$ & $66(93.0 \%)$ & $<0.001$ \\
\hline Altered mentation & $155(50.7 \%)$ & $14(34.1 \%)$ & $100(51.5 \%)$ & $41(57.7 \%)$ & 0.05 \\
\hline Baseline SOFA & $0.0(0.0-0.0)$ & $0.0(0.0-1.5)$ & $0.0(0.0-0.0)$ & $0.0(0.0-0.0)$ & 0.08 \\
\hline SOFA & $5.0(3.0-8.0)$ & $1.0(0.5-2.5)$ & $5.0(3.0-7.0)$ & $9.0(7.0-11.0)$ & $<0.001$ \\
\hline Delta SOFA & $5.0(2.0-8.0)$ & $1.0(0.0-1.0)$ & $4.0(3.0-7.0)$ & $9.0(7.0-10.0)$ & $<0.001$ \\
\hline Focus of infection & & & & & 0.01 \\
\hline Respiratory & $133(43.5 \%)$ & $18(43.9 \%)$ & $97(50.0 \%)$ & $18(25.4 \%)$ & \\
\hline Hepatobiliary & $52(17.0 \%)$ & $3(7.3 \%)$ & $31(16.0 \%)$ & $18(25.4 \%)$ & \\
\hline Gastrointestinal & $32(10.5 \%)$ & $7(17.1 \%)$ & $16(8.2 \%)$ & $9(12.7 \%)$ & \\
\hline Urinary & $24(7.8 \%)$ & $3(7.3 \%)$ & $16(8.2 \%)$ & $5(7.0 \%)$ & \\
\hline Others & $65(21.2 \%)$ & $10(24.4 \%)$ & $34(17.5 \%)$ & $21(29.6 \%)$ & \\
\hline \multicolumn{6}{|l|}{ Initial vital signs } \\
\hline $\begin{array}{l}\text { Systolic blood pressure, } \\
\mathrm{mmHg}\end{array}$ & $93.0(83.0-100.0)$ & $98.0(93.0-103.0)$ & $95.0(85.8-111.3)$ & $83.0(70.0-92.0)$ & $<0.001$ \\
\hline $\begin{array}{l}\text { Diastolic blood pressure, } \\
\mathrm{mmHg}\end{array}$ & $59.0(50.0-67.0)$ & $63.0(55.5-70.5)$ & $60.0(52.8-68.3)$ & $51.0(38.0-57.0)$ & $<0.001$ \\
\hline $\begin{array}{l}\text { Respiratory } \\
\text { breaths/min }\end{array}$ & $24.0(22.0-26.0)$ & $24.0(22.0-28.0)$ & $24.0(22.0-26.0)$ & $24.0(22.0-28.0)$ & 0.38 \\
\hline Pulse rate, beats/min & $106.5(26.6)$ & $101.8(31.7)$ & $105.2(24.6)$ & $112.6(27.9)$ & 0.07 \\
\hline Body temperature, ${ }^{\circ} \mathrm{C}$ & $37.5(36.6-38.5)$ & $37.8(36.8-38.7)$ & $37.4(36.6-38.4)$ & $37.5(36.5-38.6)$ & 0.76 \\
\hline Initial lactic acid, mmol/L & $2.0(1.1-3.5)$ & $1.4(1.0-2.4)$ & $1.6(1.0-2.5)$ & $4.2(2.6-6.8)$ & $<0.001$ \\
\hline
\end{tabular}

Time to the bundle implementation

\begin{tabular}{lccccc}
$\begin{array}{l}\text { Serum lactate measurement, } \\
\text { min }\end{array}$ & $14.5(11.0-25.0)$ & $15.0(12.0-28.0)$ & $15.0(11.0-25.0)$ & $13.0(9.0-20.0)$ & 0.02 \\
\hline Blood cultures, min & $78.0(46.0-146.0)$ & $80.0(41.0-169.0)$ & $83.0(49.0-162.5)$ & $61.0(35.0-107.0)$ & 0.01 \\
Broad-spectrum antibiotics & $143.0(88.0-236.0)$ & $190.5(128.8-350.0)$ & $151.0(89.5-263.8)$ & $108.0(78.5-147.0)$ & $<0.001$ \\
\hline 3-hour bundle compliance & $165(53.9)$ & $13(31.7)$ & $96(49.5)$ & $56(78.9)$ & $<0.001$ \\
\hline 30-day mortality & $71(23.2)$ & $5(12.2)$ & $43(22.2)$ & $23(32.4)$
\end{tabular}

All values are presented as median (interquartile range), mean (standard deviation) or number (percent), as appropriate. qSOFA, quick sequential organ failure assessment; SOFA, sequential organ failure assessment.

bundle compliance were analysed using univariable logistic regression. The variables with entry-level $P$-values $<0.10$ in the univariable analysis were selected for multivariable stepwise logistic regression analysis with the backward elimination method. The results of the logistic regression analysis are presented as odds ratios (ORs) and 95\% confidence inter- vals (CIs). Models were tested for goodness of fit using the Hosmer-Lemeshow method. Two-sided $P$-values $<0.05$ were considered statistically significant. All statistical analyses were performed using SPSS Statistics for Macintosh, version 20.0 (IBM Corp., Armonk, NY, USA). Assuming the baseline 3-hour bundle compliance rate before CDSS of $40 \%$, a sample 
TA B L E 2. Comparison of baseline characteristics between the sepsis patients before and after the routine implementation of the qSOFA-based sepsis decision support system.

\begin{tabular}{|c|c|c|c|c|}
\hline Characteristic & Overall $(n=265)$ & CDSS group $(\mathrm{n}=152)$ & Control group $(\mathrm{n}=113)$ & $P$-value \\
\hline Age, years & $69.0(58.0-77.0)$ & $70.0(60.0-78.8)$ & $66.0(55.0-76.0)$ & 0.08 \\
\hline Male & $156(58.9 \%)$ & $91(59.9 \%)$ & $65(57.5 \%)$ & 0.70 \\
\hline Charlson Comorbidity Index & $5.0(4.0-7.0)$ & $5.0(4.0-7.0)$ & $6.0(3.5-8.0)$ & 0.17 \\
\hline qSOFA & & & & 0.47 \\
\hline 2 & $232(87.5 \%)$ & $135(88.8 \%)$ & $97(85.8 \%)$ & \\
\hline 3 & $33(12.5 \%)$ & $17(11.2 \%)$ & $16(14.2 \%)$ & \\
\hline \multicolumn{5}{|l|}{ qSOFA component } \\
\hline Respiratory rate $\geq 22 / \mathrm{min}$ & $223(84.2 \%)$ & $123(80.9 \%)$ & $100(88.5 \%)$ & 0.10 \\
\hline $\begin{array}{l}\text { Systolic blood pressure }<100 \\
\mathrm{mmHg}\end{array}$ & $201(75.8 \%)$ & $112(73.7 \%)$ & $89(78.8 \%)$ & 0.34 \\
\hline Altered mentation & $141(53.2 \%)$ & $87(57.2 \%)$ & $54(47.8 \%)$ & 0.13 \\
\hline Baseline SOFA & $0.0(0.0-0.0)$ & $0.0(0.0-0.8)$ & $0.0(0.0-0.0)$ & 0.06 \\
\hline SOFA & $6.0(4.0-9.0)$ & $6.0(4.0-9.0)$ & $6.0(4.0-8.0)$ & 0.30 \\
\hline Delta SOFA & $6.0(3.0-8.0)$ & $6.0(3.0-9.0)$ & $5.0(4.0-8.0)$ & 0.53 \\
\hline Focus of infection & & & & 0.71 \\
\hline Respiratory & $115(43.4 \%)$ & $65(42.8 \%)$ & $50(44.2 \%)$ & \\
\hline Hepatobiliary & $49(18.5 \%)$ & $26(17.1 \%)$ & $23(20.4 \%)$ & \\
\hline Gastrointestinal & $25(9.4 \%)$ & $14(9.2 \%)$ & $11(9.7 \%)$ & \\
\hline Urinary & $21(7.9 \%)$ & $15(9.9 \%)$ & $6(5.3 \%)$ & \\
\hline Others & $55(20.8 \%)$ & $32(21.1 \%)$ & $23(20.4 \%)$ & \\
\hline \multicolumn{5}{|l|}{ Initial vital signs } \\
\hline Systolic blood pressure, $\mathrm{mmHg}$ & $92.0(81.0-100.0)$ & $92.5(81.3-104.8)$ & $91.0(81.0-100.0)$ & 0.66 \\
\hline Diastolic blood pressure, $\mathrm{mmHg}$ & $58.0(49.5-66.5)$ & $58.0(50.0-67.8)$ & $58.0(49.0-65.5)$ & 0.58 \\
\hline Respiratory rate, breaths/min & $24.0(22.0-26.0)$ & $24.0(22.0-26.0)$ & $24.0(22.0-26.0)$ & 0.82 \\
\hline Pulse rate, beats/min & $107.2(25.7)$ & $105.8(27.7)$ & $109.1(22.6)$ & 0.30 \\
\hline Body temperature, ${ }^{\circ} \mathrm{C}$ & $37.4(36.6-38.5)$ & $37.3(36.5-38.2)$ & $37.5(36.8-38.7)$ & 0.15 \\
\hline ED occupancy rate, $\%$ & $133.3(106.9-162.8)$ & $123.9(98.9-143.5)$ & $156.2(131.1-175.2)$ & $<0.001$ \\
\hline Initial lactic acid, mmol/L & & & & 0.18 \\
\hline$<2$ & $125(47.2 \%)$ & $79(52.0 \%)$ & $46(40.7 \%)$ & \\
\hline $2-4$ & $82(30.9 \%)$ & $44(28.9 \%)$ & $38(33.6 \%)$ & \\
\hline$>4$ & $58(21.9 \%)$ & $29(19.1 \%)$ & $29(25.7 \%)$ & \\
\hline Disease severity & & & & 0.72 \\
\hline Sepsis & $194(73.2 \%)$ & $110(72.4 \%)$ & $84(74.3 \%)$ & \\
\hline Septic shock & $71(26.8 \%)$ & $42(27.6 \%)$ & $29(35.7 \%)$ & \\
\hline Culture-positive sepsis & $62(24.2 \%), n=256$ & $39(27.1 \%), n=144$ & $23(20.5 \%), n=112$ & 0.23 \\
\hline
\end{tabular}

Time to the bundle implementa-

tion

Serum lactate measurement, $14.0(11.0-23.0), \mathrm{n}=264 \quad 14.0(11.0-22.0), \mathrm{n}=151 \quad 14.0(10.0-25.0), \mathrm{n}=113 \quad 0.95$ $\min$

Blood cultures, $\min \quad 77.5(46.0-141.8), \mathrm{n}=256 \quad 77.5(44.5-139.8), \mathrm{n}=144 \quad 76.5(46.5-145.5), \mathrm{n}=112 \quad 0.90$ Broad-spectrum antibiotics, min $136.0(85.0-230.0), \mathrm{n}=255139.0(90.5-257.0), \mathrm{n}=144132.0(84.0-208.0), \mathrm{n}=1110.22$ Gap time from blood culture to $34.0(5.3-85.0), \mathrm{n}=248 \quad 40.0(11.8-91.3), \mathrm{n}=138 \quad 20.0(2.8-71.8), \mathrm{n}=110 \quad 0.008$ antibiotics. min 
TA B L E 2. Continued.

\begin{tabular}{lcccc} 
Characteristic & Overall $(\mathrm{n}=265)$ & CDSS group $(\mathrm{n}=152)$ & Control group $(\mathrm{n}=113)$ & $P$-value \\
Compliance with bundle & & & & \\
Serum lactate measurement & $260(98.1 \%)$ & $150(98.7 \%)$ & $110(97.3 \%)$ & 0.65 \\
Blood cultures & $214(80.8 \%)$ & $119(78.3 \%)$ & $95(84.1 \%)$ & 0.24 \\
\hline Broad-spectrum antibiotics & $163(61.5 \%)$ & $89(58.6 \%)$ & $74(65.5 \%)$ & 0.25 \\
\hline Fluid administration* & $265(100 \%)$ & $152(100 \%)$ & $113(100 \%)$ & - \\
\hline All compliance & $152(57.4 \%)$ & $80(52.6 \%)$ & $72(63.7 \%)$ & 0.07 \\
\hline $30-$ day mortality & $66(24.9 \%)$ & $41(27.0 \%)$ & $25(22.1 \%)$ & 0.37 \\
\hline
\end{tabular}

All values are presented as median (interquartile range), mean (standard deviation) or number (percent), as appropriate.

* The 25 patients with severe heart failure or end-stage renal disease were administered $20 \mathrm{~mL} / \mathrm{kg}$ of intravenous crystalloid and were categorized in the completion of the fluid administration.

CDSS, clinical decision support system; qSOFA, quick sequential organ failure assessment; SOFA, sequential organ failure assessment.

size of 190 patients with sepsis was calculated based on $80 \%$ power, 0.05 alpha, and a 0.20 proportional increase.

\section{Results}

Between July 1 and December 31, 2016, there were 924 adult patients with qSOFA scores $\geq 2$ upon arrival at our ED (Fig. 2). Of these cases, 618 were excluded from the study because the patients either were not suspected of having an infectious disease $(n=534)$, refused further examination and treatment (including those with "do not resuscitate" orders) (n $=66$ ), or were directly transferred to another hospital from the ED within 3 hours $(n=18)$. A total of 306 cases were finally included in this study and grouped according to disease severity: infectious disease $(\mathrm{n}=41,13.4 \%)$, sepsis $(\mathrm{n}=194$, $63.4 \%)$, and septic shock $(\mathrm{n}=71,23.2 \%)$.

Table 1 summarises the baseline clinical characteristics and outcomes of the patients according to the severity of the infection. The study patients predominantly had qSOFA scores of 2 $(85.6 \%)$, whereas the proportion of patients with qSOFA scores of 3 was significantly higher among patients in the septic shock group $(22.5 \%)$. As disease severity increased (from infectious disease to septic shock), the SOFA score, serum lactic acid level, and 3-hour resuscitation bundle compliance rate significantly increased, whereas the time to bundle implementation time decreased.

The baseline characteristics and comparisons of the beforeand-after CDSS analysis for patients with sepsis and septic shock development are presented in Table 2. There were no statistically significant differences between the CDSS and control groups in terms of suspected infection focus, disease severity, SOFA score or culture-positive sepsis. The median ED occupancy rate during the study period was $133.3 \%$ (IQR, $106.9 \%-162.8 \%$ ), and it was significantly higher in the control group (median, $123.9 \%$ vs. $156.2 \%$; $P<0.001$ ). The 3 hour resuscitation bundle compliance for all 4 components was $57.4 \%$, with no significant difference between the groups.

Among the 62 culture-positive patients, Escherichia coli $(\mathrm{n}=26,9.8 \%)$ was most frequently identified microorganism in blood culture, followed by and Klebsiella pneumoniae $(\mathrm{n}=14,5.3 \%)$ and Staphylococcus aureus $(\mathrm{n}=6,2.3 \%)$ (Supplement Table 1). The broad-spectrum antimicrobial agent was administered for 255 patients during ED stay. Piperacillin/tazobactam $(\mathrm{n}=86,33.7 \%)$ and ceftriaxone $(\mathrm{n}=$ $54,21.2 \%$ ) were the most frequently administered antibiotics in our study (Supplement Table 2).

In the univariable analysis, 3-hour resuscitation bundle compliance was significantly more likely with increases in patient age (OR, 1.024; 95\% CI, 1.006-1.042; $P=0.009$ ), respiratory rate (OR, $1.063 ; 95 \% \mathrm{CI}, 1.010-1.118 ; P=0.019)$, pulse rate (OR, 1.010; 95\% CI, 1.001-1.020; $P=0.038$ ), and body temperature (OR, $1.611 ; 95 \% \mathrm{CI}, 1.306-1.987 ; P<0.001)$. SOFA score, ED occupancy rate, and the presence of the CDSS were not significantly associated with 3-hour resuscitation bundle compliance (Table 3). The multivariable logistic regression analysis indicated that age (AOR [adjusted odds ratio], 1.033; 95\% CI, 1.012-1.053; $P=0.002$ ) and body temperature (AOR, $1.677 ; 95 \% \mathrm{CI}, 1.339-2.100 ; P<0.001$ ) were independently associated with 3-hour resuscitation bundle compliance among sepsis and septic shock patients with qSOFA scores $\geq 2$ upon arrival at our ED. The CDSS did not affect the compliance with the 3-hour resuscitation bundle standard (AOR, 0.591; 95\% CI, $0.342-1.021 ; P=0.059$ ).

\section{Discussion}

We found that qSOFA score-based sepsis CDSS did not affect 3-hour resuscitation bundle compliance for the patients with qSOFA scores $\geq 2$ upon arrival at our ED who developed sepsis and septic shock while in the ED. Among the patients with qSOFA scores $\geq 2$ upon arrival at our ED, who were suspected of having an infectious disease, $86.6 \%(265 / 306)$ developed sepsis and septic shock while in the ED. For patients who developed sepsis or septic shock, the 3-hour resuscitation bundle compliance rate was $61.5 \%$, and administering broadspectrum intravenous antibiotics was the main barrier to bundle compliance among the bundle elements. Age and body temperature at ED arrival were the only independent factors that significantly influenced 3-hour resuscitation bundle compliance, while ED occupancy rate did not affect 3-hour resuscitation 
T A B L E 3. Univariable and multivariable logistic regression analyses of 3-hour resuscitation bundle compliance for sepsis patients with positive qSOFA scores upon emergency department admission.

Univariable analysis

Multivariable analysis

Odds ratio 95\% Confidence interval $P$-value Adjusted odds ratio 95\% Confidence interval $P$-value

Charlson Comorbidity Index $\quad 1.06 \quad 0.97-1.17 \quad 0.21$

qSOFA score

$\begin{array}{lccc}2 & \text { Reference } & & \\ 3 & 1.57 & 0.73-3.39 & 0.25\end{array}$

qSOFA component

Respiratory rate $>22 / \mathrm{min}$

$1.13 \quad 0.58-2.20 \quad 0.71$

Systolic blood pressure $<0.90 \quad 0.51-1.59 \quad 0.71$

$100 \mathrm{mmHg}$

$\begin{array}{llllll}\text { Altered mentation } & 1.21 & 0.75-1.98 & 0.44 & & \\ \text { Baseline SOFA } & 0.99 & 0.79-1.24 & 0.92 & & \\ \text { SOFA } & 1.06 & 0.98-1.14 & 0.13 & & 0.99-1.17\end{array}$

Focus of infection

$\begin{array}{lccc}\text { Respiratory } & \text { Reference } & & \\ \text { Hepatobiliary } & 1.01 & 0.52-2.04 & 0.85 \\ \text { Gastrointestinal } & 1.75 & 0.70-4.39 & 0.23 \\ \text { Urinary } & 1.65 & 0.62-4.39 & 0.32 \\ \text { Others } & 1.07 & 0.56-2.04 & 0.85\end{array}$

Initial vital signs

\begin{tabular}{|c|c|c|c|c|c|c|}
\hline $\begin{array}{l}\text { Systolic blood pressure, } \\
\mathrm{mmHg}\end{array}$ & 1.00 & $0.99-1.01$ & 0.99 & & & \\
\hline $\begin{array}{l}\text { Diastolic blood pressure, } \\
\mathrm{mmHg}\end{array}$ & 1.00 & $0.98-1.01$ & 0.73 & & & \\
\hline $\begin{array}{l}\text { Respiratory } \\
\text { breaths/min }\end{array}$ & 1.06 & $1.01-1.12$ & 0.02 & 1.06 & $1.00-1.12$ & 0.05 \\
\hline Pulse rate, beats/min & 1.01 & $1.00-1.02$ & 0.04 & & & \\
\hline Body temperature, ${ }^{\circ} \mathrm{C}$ & 1.61 & $1.31-1.99$ & $<0.001$ & 1.68 & $1.34-2.10$ & $<0.001$ \\
\hline ED occupancy rate, $\%$ & 1.00 & $0.99-1.00$ & 0.29 & & & \\
\hline \multicolumn{7}{|l|}{ Initial lactic acid, mmol/L } \\
\hline$<2$ & eference & & & & & \\
\hline $2-4$ & 1.96 & $1.10-3.49$ & 0.02 & & & \\
\hline$>4$ & 1.66 & $0.88-3.14$ & 0.12 & & & \\
\hline $\begin{array}{l}\text { qSOFA score-based decision } \\
\text { system }\end{array}$ & 0.63 & $0.38-1.04$ & 0.07 & 0.59 & $0.34-1.02$ & 0.06 \\
\hline
\end{tabular}

SOFA, sequential organ failure assessment; qSOFA, quick sequential organ failure assessment.

bundle compliance.

Sepsis and septic shock are time-dependent diseases, for which early recognition and treatment, represented by 3 -hour and 6-hour sepsis resuscitation bundles, are associated with improved outcomes $[5,12,13]$. However, bundle compliance rates for sepsis patients have generally been dismal in clinical practice, with estimates ranging from $7 \%$ to $27 \%$ [4, 12-14].
The main barriers to bundle compliance are delayed recognition by inexperienced physicians or ED resource shortages due to overcrowded ED environments [4, 12-14]. During the study period, the median ED occupancy rate was $133.3 \%$, which is generally recognised as overcrowding [14, 15]. However, our ED's 3-hour bundle compliance rate was $57.4 \%$, with the administration of broad-spectrum antibiotics within 3 hours 
being accomplished for $61.5 \%$ of the patients. Consistent with previous studies, a delay in the administration of broadspectrum antibiotics was the main obstacle [4, 12, 14]. The implementation of an automated dispensing cabinet in the ED would be a component of the multifaceted, hospital-wide approach to achieving prompt antibiotic administration for sepsis patients $[16,17]$.

Despite some controversy regarding the prognostic value of the qSOFA score [18-21], 86.6\% of patients with qSOFA scores $\geq 2$ at ED presentation developed sepsis or septic shock while in the ED. Our findings suggest that qSOFA score when patients arrive at the ED may be a reliable screening strategy for identifying patients at risk of sepsis, and ED physicians and nurses should act promptly for patients with qSOFA scores $\geq$ 2. However, our qSOFA-based CDSS was not associated with an improvement in bundle compliance in this study. Some limitations of our system would contribute to this unimprovement. Firstly, the system only consisted of displaying of an "S" beside the patient's name, 3-hour and 6-hour bundle checklists, and a SOFA score calculator to minimise alert fatigue and clinician workflow interruptions. However, this non-interrupting alert system might fail to arouse physicians' attention and consequently fail to improve bundle compliance. Also, the ED physicians would be highly likely to identify the patients with qSOFA scores $\geq 2$ as high acuity patients without the CDSS. Secondly, the clinicians and nurses could recognise patients with qSOFA scores $\geq 2$ via the ED patient list, but clinicians are required to open the program to view the checklist and be required to manually input some laboratory and clinical values to use the SOFA score calculator. Thirdly, the bundle compliance rate in our ED was higher (57.4\%) than previously reported rates $(<30 \%)$, and the possible positive impact could be obscured.

We found that older age and higher body temperature at ED arrival were associated with 3-hour bundle compliance. Other vital signs, ED crowding, or the application of a qSOFA scorebased CDSS showed no significant association with bundle compliance in the multivariable logistic regression analysis. Elderly patients or those with higher body temperatures were given high priority among those with a high risk of developing sepsis upon ED arrival, regardless of ED overcrowding or the presence of the CDSS program. These results suggest that physicians and nurses gave priority to the patients who seemed to have a higher risk of deterioration. Many artificial intelligence-based early warning systems and prediction models are developed in critical care medicine, including sepsis, acute respiratory distress syndrome, heart failure, and inhospital cardiac [22-24], which demonstrated the outperformance compared to conventional methods in prediction. A more precise early warning system identifying the patients in order of the risk of deterioration due to sepsis in ED and an automated alarm system would improve the sepsis management and patients' outcome. Also, it would enhance accurate decision-making and efficacy in allocations of medical resources.

Our study had several limitations. Firstly, we developed and applied the system at a single medical centre and included a small number of patients during a short study period. This limits our findings' generalisability to other institutions or patient populations, and our results should be cautiously interpreted, especially when considering EDs with low levels of crowding. Secondly, although we prospectively collected the data after the routine implementation of the CDSS, we also retrospectively collected the data from before the CDSS implementation. Thirdly, all clinicians treated patients according to the SSC guidelines, but we could not adjust for all potential inter-clinician variability and the effects of unobserved biases. Finally, we only determined qSOFA scores at ED arrival instead of using real-time bedside monitoring.

\section{Conclusions}

In conclusion, we developed a qSOFA-based sepsis CDSS to improve the early recognition and timely treatment of sepsis, represented by 3-hour bundle compliance, but the system was not associated with improved bundle compliance. We believe that the relatively high rate of bundle compliance and overcrowding in our ED might have blunted the potential benefits of the qSOFA-based CDSS, and multifactorial, hospital-wide approaches are warranted to improve the care of patients with sepsis.

\section{AUTHOR CONTRIBUTIONS}

YJK and JHL designed the study. YJK, JHL and SWL collected the data. YJK and WYK analyzed the data. YJK analyzed the results and drafted the manuscript. All authors contributed substantially to the revision.

\section{ETHICS APPROVAL AND CONSENT TO PARTICIPATE}

Asan Medical Center's institutional review board approved the study (Study No. 2016-0818) and waived the requirement for informed consent because the study involved the analysis of a case registry. The research is in accordance with the Helsinki Declaration of 1975, as revised in 2010.

\section{ACKNOWLEDGMENT}

The authors would like to express our gratitude to all those who helped me during the writing of this manuscript, and we thank to all the peer reviewers for their opinions and suggestions.

\section{FUNDING}

This research was supported by the Basic Science Research Program, through the National Research Foundation of Korea (NRF), funded by the Ministry of Education (No. NRF2015R1D1A1A01057091).

\section{CONFLICT OF INTEREST}

The authors declare that there is no conflict of interest regarding the publication of this article. 


\section{SUPPLEMENTARY MATERIAL}

Supplementary material associated with this article can be found, in the online version, at https://oss. signavitae. com/mre-signavitae/article/1391673656692621312/ attachment/Supplementary\%20material.rar.

\section{DATA AVAILABILITY}

The data used to support the findings of this study are available from the corresponding author upon request.

\section{REFERENCES}

[1] Seymour CW, Liu VX, Iwashyna TJ, Brunkhorst FM, Rea TD, Scherag A, et al. Assessment of clinical criteria for sepsis: for the Third International Consensus Definitions for Sepsis and Septic Shock (Sepsis-3). The Journal of the American Medical Association. 2016; 315: 762-774.

[2] Singer M, Deutschman CS, Seymour CW, Shankar-Hari M, Annane $\mathrm{D}$, Bauer $\mathrm{M}$, et al. The third international consensus definitions for sepsis and septic shock (Sepsis-3). The Journal of the American Medical Association. 2016; 315: 801-810.

[3] Rhodes A, Evans LE, Alhazzani W, Levy MM, Antonelli M, Ferrer R, et $a l$. Surviving sepsis campaign: international guidelines for management of sepsis and septic shock: 2016. Intensive Care Medicine. 2017; 43: 304-377.

[4] Leisman DE, Doerfler ME, Ward MF, Masick KD, Wie BJ, Gribben JL, et al. Survival benefit and cost savings from compliance with a simplified 3-hour sepsis bundle in a series of prospective, multisite, observational cohorts. Critical Care Medicine. 2017; 45: 395-406.

[5] Pruinelli L, Westra BL, Yadav P, Hoff A, Steinbach M, Kumar V, et al. Delay within the 3-hour surviving sepsis campaign guideline on mortality for patients with severe sepsis and septic shock. Critical Care Medicine. 2018; 46: 500-505.

[6] Kawamoto K, Houlihan CA, Balas EA, Lobach DF. Improving clinical practice using clinical decision support systems: a systematic review of trials to identify features critical to success. British Medical Journal. 2005; 330: 765 .

[7] Handel DA, Wears RL, Nathanson LA, Pines JM. Using information technology to improve the quality and safety of emergency care. Academic Emergency Medicine. 2011; 18: e45-e51.

[8] Osheroff JA, Teich JM, Middleton B, Steen EB, Wright A, Detmer DE. A roadmap for national action on clinical decision support. Journal of the American Medical Informatics Association. 2007; 14: 141-145.

[9] Nelson JL, Smith BL, Jared JD, Younger JG. Prospective trial of real-time electronic surveillance to expedite early care of severe sepsis. Annals of Emergency Medicine. 2011; 57: 500-504.

[10] McNarry AF, Goldhill DR. Simple bedside assessment of level of consciousness: comparison of two simple assessment scales with the Glasgow Coma scale. Anaesthesia. 2004; 59: 34-37.

[11] Kim Y, Kang J, Ryoo SM, Ahn S, Huh JW, Kim WY. Platelet-lymphocyte ratio after granulocyte colony stimulating factor administration: an early prognostic marker in septic shock patients with chemotherapy-induced febrile neutropenia. Shock. 2019; 52: 160-165.
[12] Deis AS, Whiles BB, Brown AR, Satterwhite CL, Simpson SQ. Threehour bundle compliance and outcomes in patients with undiagnosed severe sepsis. Chest. 2018; 153: 39-45.

[13] Kang MJ, Shin TG, Jo IJ, Jeon K, Suh GY, Sim MS, et al. Factors influencing compliance with early resuscitation bundle in the management of severe sepsis and septic shock. Shock. 2012; 38: 474479.

[14] Shin TG, Jo IJ, Choi DJ, Kang MJ, Jeon K, Suh GY, et al. The adverse effect of emergency department crowding on compliance with the resuscitation bundle in the management of severe sepsis and septic shock. Critical Care. 2013; 17: R224.

[15] McCarthy ML, Aronsky D, Jones ID, Miner JR, Band RA, Baren JM, et al. The emergency department occupancy rate: a simple measure of emergency department crowding? Annals of Emergency Medicine. 2008; 51: $15-24$

[16] Sikka R, Sweis R, Kaucky C, Kulstad E. Automated dispensing cabinet alert improves compliance with obtaining blood cultures before antibiotic administration for patients admitted with pneumonia. Joint Commission Journal on Quality and Patient Safety. 2012; 38: 224-228.

[17] Cottney A. Improving the safety and efficiency of nurse medication rounds through the introduction of an automated dispensing cabinet. BMJ Quality Improvement Reports. 2014; 3: u204237.w1843.

[18] Freund Y, Lemachatti N, Krastinova E, Van Laer M, Claessens Y, Avondo A, et al. Prognostic accuracy of sepsis-3 criteria for in-hospital mortality among patients with suspected infection presenting to the emergency department. The Journal of the American Medical Association. 2017; 317: 301-308.

[19] Fernando SM, Tran A, Taljaard M, Cheng W, Rochwerg B, Seely AJE, et al. Prognostic accuracy of the quick sequential organ failure assessment for mortality in patients with suspected infection: a systematic review and meta-analysis. Annals of Internal Medicine. 2018; 168: 266-275.

[20] Lamontagne F, Harrison DA, Rowan KM. QSOFA for identifying sepsis among patients with infection. The Journal of the American Medical Association. 2017; 317: 267-268.

[21] Hwang SY, Jo IJ, Lee SU, Lee TR, Yoon H, Cha WC, et al. Low accuracy of positive qSOFA criteria for predicting 28-day mortality in critically ill septic patients during the early period after emergency department presentation. Annals of Emergency Medicine. 2018; 71: 1-9.e2.

[22] Jentzer JC, Kashou AH, Lopez-Jimenez F, Attia ZI, Kapa S, Friedman PA, et al. Mortality risk stratification using artificial intelligence-augmented electrocardiogram in cardiac intensive care unit patients. European Heart Journal Acute Cardiovascular Care. 2020; zuaa021.

[23] Zhang Z, Navarese EP, Zheng B, Meng Q, Liu N, Ge H, et al. Analytics with artificial intelligence to advance the treatment of acute respiratory distress syndrome. Journal of Evidence-Based Medicine. 2020; 13: 301312.

[24] Cho K, Kwon O, Kwon J, Lee Y, Park H, Jeon K, et al. Detecting patient deterioration using artificial intelligence in a rapid response system. Critical Care Medicine. 2020; 48: e285-e289.

How to cite this article: Youn-Jung Kim, Jae-Ho Lee, SangWook Lee, Won Young Kim. Use of quick sequential organ failure assessment score-based sepsis clinical decision support system may be helpful to predict sepsis development. Signa Vitae. 2021;17(5):86-94. doi:10.22514/sv.2021.082. 